\section{SíNDROME CORONARIANA AGUDA COMO APRESENTAÇÃO ATÍPICA DO FENÔMENO DE ROUBO SUBCLÁVIO}

\author{
ACUTE CORONARY SYNDROME AS AN ATYPICAL PRESENTATION OF \\ THE PHENOMENON OF SUBCLAVIAN STEAL
}

\begin{abstract}
Gustavo Neves de Araújo ${ }^{1,2}$, Guilherme Pinheiro Machado ${ }^{1,3}$, Luiz Carlos Corsetti Bergoli ${ }^{2}$, Felipe Homem Valle ${ }^{1,2}$, Ana Carolina Martins Mazzuca², Rodrigo Vugman Wainstein², Marco Vugman Wainstein ${ }^{1,2,3}$
\end{abstract}

\section{RESUMO}

Roubo coronariano da subclávia (RCS) é um fenômeno raro que ocorre em pacientes submetidos a cirurgia de revascularização miocárdica com enxerto de artéria torácica interna esquerda (ATIE) e que causa estenose da artéria subclávia ipsilateral e proximal à origem da ATIE. Relatamos o caso de um paciente masculino de 65 anos que apresentou quadro atípico de síndrome do RCS, manifestando-se como síndrome coronariana aguda no pós-operatório de cirurgia vascular.

Palavras-chave: Síndrome coronariana aguda; angioplastia; síndrome do roubo coronário-subclávio

\section{ABSTRACT}

Coronary subclavian steal (CSS) is a rare phenomenon that occurs in patients undergoing coronary artery bypass graft surgery using the left internal thoracic artery (LITA) causing stenosis of the ipsilateral subclavian artery proximal to the origin of the LITA. We report the case of a 65-year-old male patient who presented with atypical CSS syndrome, manifesting as acute coronary syndrome after vascular surgery.

Keywords: Acute coronary syndrome; angioplasty; coronary subclavian steal syndrome

O fenômeno do roubo coronariano da subclávia (RCS) ocorre em pacientes previamente submetidos a cirurgia de revascularização miocárdica (CRM) com enxerto de artéria torácica interna esquerda (ATIE) em que há estenose hemodinamicamente significativa da artéria subclávia ipsilateral e proximal à origem da ATIE. Nesses casos, o fluxo da ATIE pode ser revertido e causar "roubo" de fluxo da circulação coronariana durante atividades com os membros superiores'.

A manifestação clínica mais comum dessa síndrome é angina, mas os pacientes podem também apresentar isquemia silenciosa, disfunção ventricular progressiva e, mais raramente, infarto do miocárdio ${ }^{2-4}$. Por ser um evento raro, com prevalência de $2,5 \%$ em pacientes submetidos a CRM, não é incomum que passe despercebida, especialmente nos casos com revascularização recente ${ }^{5,6}$.

Relatamos um caso incomum de síndrome do RCS em paciente que apresentou síndrome coronariana aguda (SCA) no pós-operatório de cirurgia vascular.

\section{RELATO DE CASO}

Paciente masculino de 65 anos internou eletivamente para realização de endarterectomia de carótida esquerda, procedimento indicado como tratamento para acidente vascular cerebral prévio associado a achado de
Clin Biomed Res. 2017;37(3):259-262

1 Programa de Pós-graduação em Ciências da Saúde: Cardiologia e Ciências Cardiovasculares, Universidade Federal do Rio Grande do Sul (UFRGS), Hospital de Clínicas de Porto Alegre. Porto Alegre, RS, Brasil.

2 Serviço de Cardiologia, Hospital de Clínicas de Porto Alegre (HCPA). Porto Alegre, RS, Brasil.

3 Faculdade de Medicina, Universidade Federal do Rio Grande do Sul (UFRGS), Porto Alegre, RS, Brasil.

Autor correspondente: Gustavo Neves de Araujo gustavon.araujo@gmail.com Hospital de Clínicas de Porto Alegre Ramiro Barcelos, 2350. 90035-903, Porto Alegre, RS, Brasil. 
estenose de $80 \%$ na carótida interna esquerda em angiotomografia arterial do pescoço. Apresentava diversas comorbidades clínicas, entre elas diabetes mellitus tipo 2, hipertensão arterial sistêmica e cardiopatia isquêmica previamente tratada com CRM em 2011, não havendo conhecimento pela equipe médica assistente da anatomia dos enxertos. No mesmo ano de 2011 havia realizado bypass femorofemoral cruzado com reintervenção em 2015. Negava angina ou claudicação de membros superiores.

Foi submetido à cirurgia proposta, tendo apresentado como intercorrência sangramento aumentado no transoperatório ( $850 \mathrm{~mL}$ ), manejado com reposição volêmica. No primeiro dia de pós-operatório, evoluiu para edema agudo de pulmão associado a dor retroesternal intensa, agravamento de alterações prévias no eletrocardiograma (alargamento de QRS e infradesnivelamento de ST em DI, AVL, V5 e V6) (Figura 1) e elevação de troponina.

Após estabilização clínica inicial, o paciente foi encaminhado para cineangiocoronariografia. No procedimento, realizado pela via radial esquerda, foram observados os seguintes achados (Figura 2A): lesão de $30 \%$ no tronco da coronária esquerda,

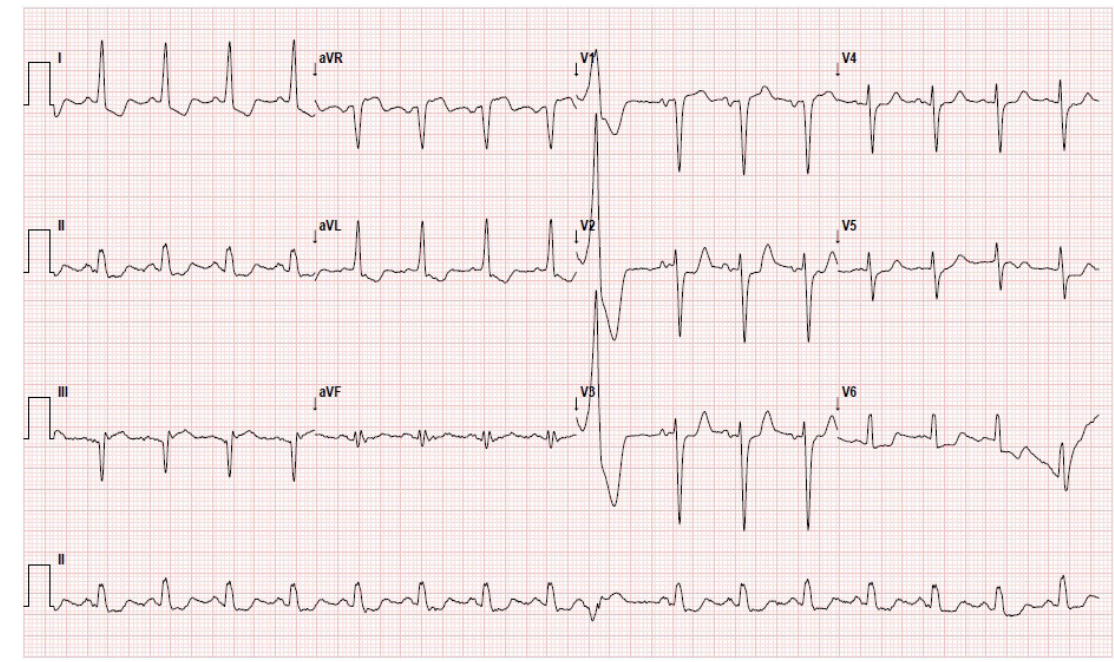

Figura 1: Alterações eletrocardiográficas. Eletrocardiograma no primeiro pós-operatório com infradesnivelamento de ST em DI, AVL, V5 e V6.

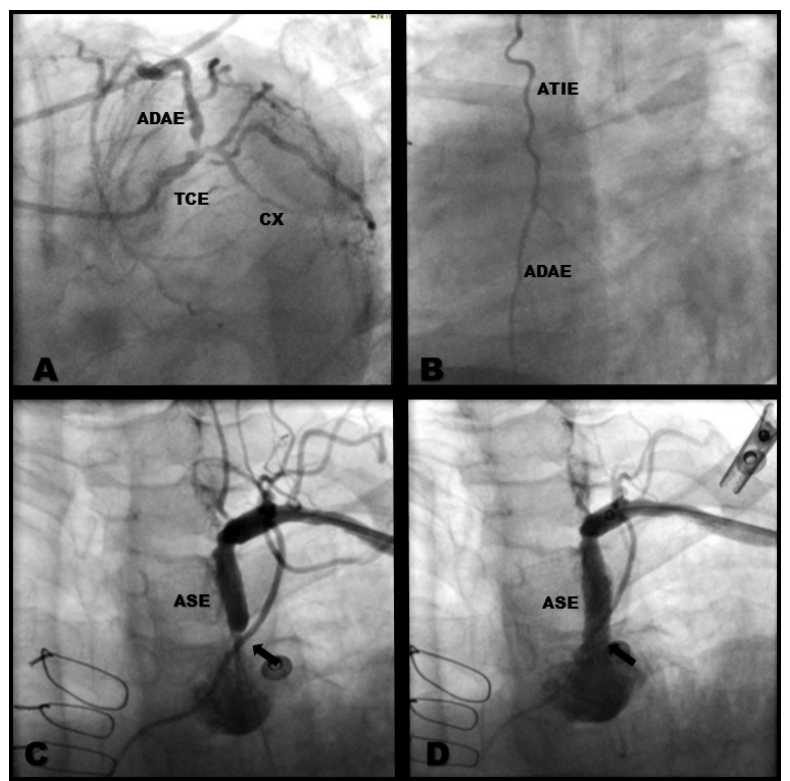

Figura 2: Alterações no cateterismo. Lesões encontradas (A); enxerto de ATIE para ADAE (B); estenose na origem da ASE e resultado após angioplastia (C, D). TCE: tronco da coronária esquerda; ADAE: artéria descendente anterior esquerda ATIE: artéria torácica interna esquerda; ASE: artéria subclávia esquerda. 
lesão de $90 \%$ no óstio da artéria descendente anterior esquerda (ADAE) com ausência de fluxo competitivo no seu leito distal, coronária circunflexa com lesão ostial de $70 \%$ e primeiro ramo marginal com lesão de $70 \%$ no seu segmento médio. A artéria coronária direita apresentava-se cronicamente ocluída com enchimento por colaterais. Enxertos venosos aortocoronarianos ocluídos. Enxerto de ATIE para ADAE (Figura 2B) apresentava-se pérvio, porém com fluxo lentificado. Durante tentativa de canulação seletiva da ATIE, observou-se estenose grave na origem da artéria subclávia esquerda que determinava redução do fluxo anterógrado na ATIE e no leito distal da ADAE (Figura $2 \mathrm{C}$ ), configurando quadro de síndrome do roubo coronariano.

Optou-se, então, por realizar angioplastia da artéria subclávia esquerda com auxílio de introdutor longo hidrofílico Destination e implante de stent autoexpansível Dynamic 8-25 mm, obtendo-se sucesso angiográfico (Figura 2D). O paciente apresentou resolução dos sintomas e recebeu alta em 48 horas.

\section{DISCUSSÃO}

A síndrome do RCS, inicialmente descrita por Harjola \& Valle em 19747 , é ocasionada por uma redução do calibre da artéria subclávia, levando à redução da pressão distal à lesão e comprometendo o fluxo sanguíneo regional, especialmente durante atividades realizadas com membros superiores ${ }^{4}$. A estenose da artéria subclávia é geralmente causada por aterosclerose, visto que o ângulo agudo entre a origem dessa artéria e a aorta ascendente aumenta o fluxo turbulento e o seu potencial aterogênico ${ }^{8}$, embora diversas outras condições possam comprometer o fluxo para esse vaso e causar roubo coronariano, tais como as arterites de Takayasu e de células gigantes. Além disso, há relatos de causas de RCS mesmo na ausência de estenose subclávia, como, por exemplo, o desenvolvimento de fluxo competitivo por fístula de hemodiálise ${ }^{1}$.

A incidência global de defeitos anatômico de artéria subclávia é difícil de ser estimada, pois os pacientes são geralmente assintomáticos, podendo apresentar sintomas cardíacos inespecíficos e isquemia silenciosa. Por esse motivo, é importante que haja um alto grau de suspeição, especialmente em pacientes que serão submetidos a CRM. Em um registro multicêntrico que avaliou 205 pacientes submetidos a cinecoronariografia e que demonstraram lesões coronarianas apropriadas para revascularização cirúrgica, foi observada prevalência de $7,8 \%$ de estenose angiográfica maior que $50 \%$ na artéria subclávia esquerda. Vale ressaltar que a diferença de pressão arterial entre os membros superiores, assim como outros sinais clínicos, apresentaram baixa acurácia para predizer estenose angiográfica ${ }^{9}$. Atualmente, alguns centros realizam rotineiramente a avaliação da artéria subclávia esquerda proximal de todos pacientes submetidos a cineangiocoronariografia, independentemente do plano cirúrgico ${ }^{6,10,11}$. Outras modalidades diagnósticas são a ultrassonografia com Doppler antes e após exercício com membro superior, angiotomografia de tórax e ressonância magnética com angiografia. Entretanto, a mensuração bilateral da pressão arterial ainda permanece um método simples para rastreamento, embora alguns achados possam passar despercebidos, especialmente os pacientes que possuem estenose bilateral ${ }^{8}$. De qualquer formar, as diretrizes do American College of Cardiology/American Heart Association (ACCF/AHA) de 2011 e da European Society of Cardiology/European Association of Cardio-Thoracic Surgery ESC/EACTS de 2014 não apresentam nenhuma definição sobre o melhor método de rastreio ${ }^{12,13}$.

Uma série de casos recente ${ }^{5}$ detectou uma prevalência de $2,5 \%$ de estenose significativa de ATIE nos pacientes submetidos a CRM. Entre os pacientes sintomáticos, a manifestação clínica mais comum é a angina, sendo rara a ocorrência de SCA. Apresentamos acima um caso de SCA sem supradesnível do segmento ST ocasionada pela redução do fluxo coronariano secundária ao roubo coronariano associado ao estresse cirúrgico, principalmente devido ao sangramento transoperatório. Dimas et al. ${ }^{10}$ reportaram caso semelhante em paciente que desenvolveu SCA com supradesnível de segmento ST após hipotensão secundária a desidratação. Existem ainda outros casos relatados com elevação do segmento ST, porém com sintomatologia presente anos após a realização do procedimento ${ }^{14,15}$.

Atualmente, o tratamento de escolha no caso de estenose ou oclusão subclávia é a angioplastia percutânea e a colocação de stent. Caso o procedimento endovascular não seja tecnicamente possível, o que pode ocorrer em até metade dos pacientes ${ }^{5}$, pode-se lançar mão da cirurgia de revascularização com bypass carotídeo-subclávia ou endarterectomia. Embora a abordagem cirúrgica possa apresentar resultados superiores em longo prazo, o risco cirúrgico pode ser proibitivo ${ }^{2}$. Em caso de impossibilidade de realização dos tratamentos acima, a realização de nova CRM surge como alternativa de maior risco. No caso relatado acima, a estenose subclávia foi facilmente acessada e tratada via artéria radial esquerda, não havendo complicações relacionadas ao procedimento imediato e em curto prazo. O paciente 
não apresentava nenhuma outra lesão com sinais inequívocos de instabilidade e a lesão culpada na SCA sem supradesnível do segmento ST pode ser difícil de ser encontrada, visto que o eletrocardiograma não é diagnóstico, mas a alteração de fluxo é um dos principais achados de lesão culpada nesse cenário. Portanto, o diagnóstico foi de fundamental importância, uma vez que o tratamento da estenose grave do óstio da artéria circunflexa exigiria, pelo ângulo de origem, implante de stent envolvendo o tronco da coronária esquerda. Essa abordagem poderia ter sido catastrófica para o paciente, já que provavelmente haveria redução adicional do fluxo da ADAE, falsamente protegida pela ATIE.
A identificação de estenose hemodinamicamente significativa da artéria subclávia previamente à CRM, bem como seu tratamento, pode prevenir a ocorrência da síndrome do RCS. Em pacientes submetidos a revascularização cirúrgica com uso de enxerto de ATIE, é necessário elevado grau de suspeição para o diagnóstico dessa condição, que, embora de rara ocorrência, pode manifestar-se como síndromes coronarianas agudas de alto risco. Além disso, os resultados do tratamento endovascular com implante de stent são excelentes, tanto em curto como em longo prazo.

\section{Conflito de interesse}

Os autores declaram não haver conflito de interesse.

\section{REFERÊNCIAS}

1. Takach TJ, Reul GJ, Cooley DA, Duncan JM, Livesay JJ, Ott DA, et al. Myocardial thievery: the coronarysubclavian steal syndrome. Ann Thorac Surg. 2006;81(1):386-92. PMid:16368420. http://dx.doi. org/10.1016/j.athoracsur.2005.05.071

2. Westerband A, Rodriguez JA, Ramaiah VG, Diethrich EB. Endovascular therapy in prevention and management of coronarysubclavian steal. $J$ Vasc Surg. 2003;38(4):699-703, discussion 704. PMid:14560215. http://dx.doi. org/10.1016/S0741-5214(03)00728-6.

3. FitzGibbon GM, Keon WJ. Coronary subclavian steal: a recurrent case with notes on detecting the threat potential. Ann Thorac Surg. 1995;60(6):18102. PMid:8787493. http://dx.doi. org/10.1016/0003-4975(95)00624-9.

4. Komenaka IK, Nguyen ET, Oyogoa SO, DeGraft-Johnson JB, Gardezi SQ. Subclavian steal syndrome in acute myocardial infarction masquerading as acute embolism to left upper extremity-a case report. Angiology. 2004;55(2):209-12. PMid:15026877. http://dx.doi.org/10.1177/00033197040 5500214.

5. Hwang HY, Kim JH, Lee W, Park $\mathrm{JH}$, Kim K-B. Left subclavian artery stenosis in coronary artery bypass: prevalence and revascularization strategies. Ann Thorac Surg. 2010;89(4):1146-50. PMid:20338322. http://dx.doi.org/10.1016/j. athoracsur.2010.01.007.
6. Takach TJ, Reul GJ, Duncan JM, Krajcer Z, Livesay JJ, Gregoric ID, et al. Concomitant brachiocephalic and coronary artery disease: outcome and decision analysis. Ann Thorac Surg. 2005;80(2):5649. PMid:16039206. http://dx.doi org/10.1016/j.athoracsur.2005.02.051.

7. Harjola PT, Valle M. The importance of aortic arch or subclavian angiography before coronary reconstruction. Chest. 1974;66(4):436-8. PMid:4416304 http://dx.doi.org/10.1378/ chest.66.4.436.

8. Cua B, Mamdani N, Halpin D, Jhamnani S, Jayasuriya S, MenaHurtado C. Review of coronary subclavian steal syndrome. J Cardiol. 2017. In press.

9. Gomes VO, Roman M, Barcellos CS, Lasevitch R, Hickmann P, Alcalde R, et al. Prevalência de estenose da artéria subclávia em pacientes candidatos a cirurgia de revascularização do miocárdio: registro multicêntrico. Rev Bras Cardiol Invasiva. 2008;16(3):307-11. http://dx.doi.org/10.1590/S217983972008000300011.

10. Dimas B, Lindsey JB, Banerjee S, Brilakis ES. ST-Segment elevation acute myocardial infarction due to severe hypotension and proximal left subclavian artery stenosis in a prior coronary artery bypass graft patient. Cardiovasc Revasc Med. 2009;10(3):191-4. PMid:19595403. http://dx.doi.org/10.1016/j. carrev.2008.08.003.
11. Elami A, Ad N, Merin G. The significance of subclavian artery injection prior to surgical myocardial revascularization. Int $\mathrm{J}$ Cardiovasc Intervent. 2003;5(2):813. PMid:12745864. http://dx.doi. org/10.1080/14628840310003271.

12. Hillis LD, Smith PK, Anderson JL, Bitt JA, Bridges CR, Byrne JG, et al, and the American College of Cardiology Foundation/American Heart Association Task Force on Practice Guidelines. 2011 ACCF/AHA guideline for coronary artery bypass graft surgery: executive summary. $J$ Thorac Cardiovasc Surg. 2017;143(1):434. PMid:22172748. http://dx.doi. org/10.1016/j.jtcvs.2011.10.015.

13. Kolh P, Windecker S. ESC/EACTS myocardial revascularization guidelines 2014. Eur Heart $J$ 2014;35(46):3235-6. PMid:25482397.

14. Tan JWC, Johan BA, Cheah FK, Wong P. Coronary subclavian steal syndrome: a rare cause of acute myocardial infarction. Singapore Med J. 2007;48(1):e5-8. PMid:17245504.

15. Mandak J, Lojik M, Tuna M, Chek JL. Coronary subclavian steal syndrome causing acute myocardial infarction in a patient undergoing coronary-artery bypass grafting. Case Rep Med. 2012;2012:798356. PMid:22969810. http://dx.doi.org/10.1155/2012/798356.

Recebido: Jan 21, 2017 Aceito: Jun 28, 2017 